\title{
Welcome to the ACNP 44th Annual Meeting
}

This issue of Neuropsychopharmacology contains the abstracts of the program of the 2005 Annual Meeting of the American College of Neuropsychopharmacology (ACNP). This is the second year that our abstracts are archived, citable, and no longer the domain only of our privileged attendees. Each year, we try to outdo ourselves in the breadth and importance of the science reported at our meeting. Having a panel accepted in the annual meeting program has gotten more competitive each year, especially, it seems, when the meeting is in Hawaii. The members of our college submitted an unprecedented number of panels and posters, and the high quality of these presentations made the work of the program committee especially difficult. Nearly two out of three proposals for panels could not be accepted because of limited space and time. Under the able chairmanship of Trey Sunderland, the program committee assembled a scientific program that will challenge and stimulate our membership and our guests. This is reflected in the abstracts in this issue of the Journal. Each year the program committee is given a charge by the president to try to emphasize an area of our field that is especially timely or that may not have received enough attention in prior years. This year I asked Dr. Sunderland to highlight aging and dementia, because the science is exploding and because these subjects generally do not receive as much attention at our annual meeting as do mainstream psychiatric disorders and substance abuse disorders. The program reflects to some degree this emphasis, maybe not as much as we had hoped given the relatively few submissions focused on these subject areas, but this area of research will be better represented than in past years. I want to express my special appreciation to Ronnie Wilkins, Kathy Latimer, Jennifer Mahar and the rest of the ACNP staff for their tireless efforts to make the meeting, the program, and this issue of the Journal exceptional and unforgettable. I believe that our program this year is especially rich and diverse. We should have a great meeting. Welcome to Hawaii and to the 2005 Annual Meeting of the ACNP.

Daniel R. Weinberger, M.D.

President

American College of Neuropsychopharmacology 


\section{Neuropsychopharmacology}

\section{American College of Neuropsychopharmacology $44^{\text {th }}$ Annual Meeting ACNP OFFICERS}

President: . . . . . . . Daniel R.Weinberger, M.D.

President-Elect: . . . . . . Kenneth L. Davis, M.D.

Secretary: . . . . . . . Oakley Ray, Ph.D.

Treasurer: . . . . . . . Irwin J. Kopin, M.D.

\section{ACNP COUNCIL}

Eric J. Nestler, M.D, Ph.D. (03-05)

William T. Carpenter, Jr., M.D. (04-06)

Michael Davis, Ph.D. (05-07)

Carol A. Tamminga, M.D. (05-06)
Ellen Frank, Ph.D. (2005)

Raquel E. Gur, M.D., Ph.D. (04-06)

Mary Jeanne Kreek, M.D. (05-07)

Dennis S. Charney, M.D. (04-05)

\section{PROGRAM AND SCIENTIFIC COMMUNICATIONS COMMITTEE}

Trey Sunderland, Chair . . . . . . . . . . 2004-06

Larry Siever, Co-Chair . . . . . . . . . 2005-07

Nancy Andreasen . . . . . . . . . . . . 2003-05

John Krystal . . . . . . . . . . . . . 2003-05

Bruce McEwen ... . . . . . . . . . . . . 2003-05

Charles Nemeroff . . . . . . . . . . . . . . . 2003-05

Daniel Pine ..................2003-05

Judith Rapoport . . . . . . . . . . . . . 2003-05

Rita Valentino ...............2003-05

Elizabeth Young . . . . . . . . . . . . . . . . . . . 2003-05

Lori Altshuler . . . . . . . . . . . . . . . 2004-06

Jay Baraban . . . . . . . . . . . . . . . . . . 2004-06

Jacqueline Crawley . . . . . . . . . . . . . . . 2004-06

Suzanne Haber . . . . . . . . . . . . . . . . 2004-06
Edward Jones . . . . . . . . . . . . . . . . 2004-06

Edythe London . . . . . . . . . . . . . . . . . 2004-06

Perry Renshaw . . . . . . . . . . . . . . . . . 2004-06

Karen Berman . . . . . . . . . . . . . . . . . . . . . 2005-07

John Csernansky . . . . . . . . . . . . . . . . . 2005-07

Steven DeKosky . . . . . . . . . . . . . . . . 2005-07

David Goldman . . . . . . . . . . . . . . . . . . 2005-07

Dilip Jeste . . . . . . . . . . . . . . . . . . . 2005-07

Pat Levitt . . . . . . . . . . . . . . . 2005-07

Helen Mayberg . . . . . . . . . . . . . . . . . 2005-07

Lorna Role . . . . . . . . . . . . . . . . . . . . . . 2005-07

Gary Small . . . . . . . . . . . . . . . . . . . . . 2005-07

George Zubenko . . . . . . . . . . . . . . . . 2005-07

Raquel Gur ................ Council Liaison

\section{Future ACNP Annual Meetings}

December 3-7, 2006

December 9-13, 2007

December 7-II, 2008
The Westin Diplomat

Boca Raton Resort and Club

The Fairmont Scottsdale Princess
Hollywood, Florida

Boca Raton, Florida

Scottsdale, Arizona

\section{American College of Neuropsychopharmacology}

Executive Office

545 Mainstream Drive, Suite 110

Nashville, TN 37228 USA

ph: $615-324-2360$

fx: 6|5-324-236|

www.acnp.org

acnp@acnp.org 